\section{AB0466 ADVERSE EVENTS AND THE RELAPSE RISK OF SYSTEMIC LUPUS ERYTHEMATOSUS DURING HYDROXYCHLOROQUINE TREATMENT}

Kunihiro Ichinose ${ }^{1}$, Keita Fujikawa ${ }^{2}$, Akinari Mizokami ${ }^{2}$, Toshiyuki Aramaki ${ }^{3}$, Yukitaka Ueki ${ }^{3}$, Mizuna Eguchi ${ }^{1}$, Momoko Okamoto ${ }^{1}$, Yushiro Endo ${ }^{1}$,

Sousuke Tsuji ${ }^{1}$, Ayuko Takatani ${ }^{1}$, Toshimasa Shimizu ${ }^{1}$, Remi Sumiyoshi ${ }^{1}$, Tomohiro Koga ${ }^{1}$, Shin-Ya Kawashiri ${ }^{1}$, Naoki Iwamoto ${ }^{1}$, Takashi Igawa ${ }^{1}$, Mami Tamai ${ }^{1}$, Hideki Nakamura ${ }^{1}$, Tomoki Origuchi ${ }^{4}$, Atsushi Kawakami ${ }^{1}$. ${ }^{1}$ Nagasaki University Graduate School of Biomedical Sciences, Department of Immunology and Rheumatology, Unit of Advanced Preventive Medical Sciences, Nagasaki, Japan; ${ }^{2} \mathrm{JCHO}$ Isahaya General Hospital, Department of Rheumatology, Isahaya, Japan; ${ }^{3}$ Sasebo Chuo Hospital, Rheumatic disease center, Sasebo, Japan; ${ }^{4}$ Nagasaki University Graduate School of Biomedical Sciences, Department of Rehabilitation Sciences, Nagasaki, Japan

Background: The antimalarial drug hydroxychloroquine (HCQ) is used worldwide to control the disease activity of systemic lupus erythematosus (SLE). In Japan, HCQ was not approved until September 2015 due to the problem of retinopathy induced by chloroquine. There is insufficient evidence of the effects of $\mathrm{HCQ}$ or adverse events linked to HCQ treatment.

Objectives: Here, we evaluated adverse events and the relapse risk of SLE during HCQ treatment.

Methods: We retrospectively analyzed the data of 109 patients diagnosed with SLE and treated with HCQ for $>12$ months at Nagasaki University Hospital and community hospitals. The demographic data included the patient's age at the onset of SLE, gender, the disease duration of SLE (the time from the diagnosis of SLE until the renal biopsy), comorbidities of Sjögren syndrome (SS)/anti-phospholipid syndrome (APS), and the treatment for induction. We identified the risk of adverse events and relapse at 12 months after the introduction of HCQ. Decision tree models predicting relapse were built with the Classification and Regression Trees (CART) algorithm. The data of the length of time between a patient's HCQ induction to the first observation of relapse was analyzed by the Kaplan-Meier method with a log-rank test.

Results: Most of the patients were female $(88.1 \%)$. The median age at the introduction of $\mathrm{HCQ}$ was 40.0 years (interquartile range [IQR] 30.550.0 years), and the SLE disease duration was 95 months (IQR 38.0184.5 months). The mean observation period after HCQ introduction was 12 months. The comorbidity rates of SS and APS were $25.7 \%$ and $20.2 \%$, respectively. The SELENA-SLEDAI scores were significantly decreased at 3 months post-HCQ introduction. The dose of oral prednisolone was significantly decreased at 6 months post-HCQ introduction. Eighty-six patients $(78.9 \%)$ were continuing $\mathrm{HCQ}$ at 12 months post-introduction. Adverse events occurred in 27 patients $(24.8 \%)$, including skin rashes $(n=11,10.1 \%)$ and gastrointestinal symptoms ( $n=6,5.5 \%)$. The sole predictive factor for adverse events was the white blood cell (WBC) count at baseline (odds ratio: $0.9997,95 \% \mathrm{Cl}$ : 0.9994-0.9999, $\mathrm{p}=0.0285$ ). Twelve of 86 patients $(14.0 \%)$ experienced relapse that required the start of prednisolone or an immunosuppressant or an increased prednisolone dose. The multivariate analysis revealed that the $\mathrm{C} 4$ value at baseline was a predictive factor of relapse (odds ratio: 0.841 , 95\% 0.984, $\mathrm{p}=0.0097$ ). The cut-off point determined by the CART algorithm showed that $\mathrm{C} 4 \geq 9.3 \mathrm{mg} / \mathrm{dl}$ at baseline provides the best performance for predicting relapse. The Kaplan-Meier analysis showed that compared to $\mathrm{C} 4<9.3 \mathrm{mg} / \mathrm{dl}$ at baseline, $\mathrm{C} 4 \geq 9.3 \mathrm{mg} / \mathrm{dl}$ at baseline was correlated with being free from relapse $(p=0.0032)$.

Conclusion: A lower C4 value at HCQ introduction was a predictive factor for the relapse of SLE, and a lower WBC count was a predictive factor for adverse events.

\section{REFERENCES}

[1] Ponticelli C, Moroni G. Hydroxychloroquine in systemic lupus erythematosus (SLE). Expert Opin Drug Saf. 2017 Mar;16(3):411-419. doi: 10.1080/ 14740338.2017.1269168.

Disclosure of Interests: None declared DOI: 10.1136/annrheumdis-2019-eular.1171

\section{AB0467 \\ IMPROVED DISEASE CONTROL OF SYSTEMIC LUPUS ERYTHEMATOSUS AND ITS POSSIBLE ASSOCIATION WITH AGGRESSIVE USE OF IMMUNOSUPPRESSANTS}

Chihiro Imaizumi, Yuki Inoue, Takaharu Kagtagiri, Sayaka Takenaka, Hideki Ito, Ayako Hirata, Takehisa Ogura, Hideto Kameda. Toho University, Division of Rheumatology, Department of Internal Medicine, Tokyo, Japan

Background: The outcome of patients with systemic lupus erythematosus (SLE) has considerably improved in recent decades.

Objectives: The aim of this retrospective study was to examine whether the disease control has been actually improved and its possible association with the altered balance between the use of immunosuppressants and glucocorticoids.

Methods: We enrolled SLE patients who visited Toho University Ohashi Medical Center during 2012-2017 (Group A, 79 patients), and compared them with patients during 1999-2003 (Group B, 68 patients; not overlapping with Group A). All the patients met the American College of Rheumatology 1997 revised criteria for SLE classification. Patient backgrounds, the dose of glucocorticoids and the use of immunosuppressants at the times of SLE onset and disease flare were reviewed from the medical records. The disease flare was defined as a new BILAG 2004 A or B score in at least one system.

Results: The age at disease onset and sex, as well as the presence of lupus nephritis and serositis were comparable between 2 groups, although central nervous system manifestations were less frequent in Group A $(7 \%$ versus $19 \%$ in Group $B, p=0.04)$. The average number of flare per person-year was significantly reduced in Group A than Group B (0.26 versus 0.4 , respectively, $p<0.01)$. In the initial treatment, the median dosage of oral prednisolone was equivalent in both groups (30 $\mathrm{mg}$ day, $\mathrm{p}=0.64$ ), while the total glucocorticoid dosage in the initial 16 weeks was significantly reduced in Group A (1,960 mg prednisolone equivalent versus $2,913 \mathrm{mg}$ in Group $\mathrm{B}, \mathrm{p}=0.01$ ). And the inclusion rate of immunosuppressants in the initial SLE treatment was significantly higher in Group A ( $43 \%$ versus $6 \%$ in Group B, $p<0.01$ ). Further, upon disease flare, the dose of prednisolone for it was significantly lower in Group A $(6.5 \mathrm{mg} /$ day versus $14 \mathrm{mg} /$ day in Group $\mathrm{B}, \mathrm{p}<0.01$ ) and the rate of introduction or alteration of immunosuppressants were significantly higher in Group A ( $81 \%$ versus $24 \%$ in Group $B, p<0.01)$. Infection rates were similar between the groups $(p=0.44)$.

Conclusion: Aggressive use of immunosuppressants in recent years resulted in the reduction of the rate of SLE flare as well as that of cumulative glucocorticoid dosage.

\section{REFERENCES}

[1] Bernatsky S, et al. Arthritis Rheum 2006; 54: 2550-7

[2] Lee $Y$, et al. Lupus 2016; 25: 727-34.

[3] Guillermo R.I. Rheumatology 2012; 51: 1145-53

[4] Ugarte A, et al. Lupus 2011; 4: 423

[5] Pepper R, et al. Nephrol Dial Transplant 2009; 24: 3717-23.

[6] Saag KG, et al. Am J Med 1994;96: 115-23

[7] Ruiz-Irastorza G, et al. Arthritis Res Ther 2009;11:R109.

Disclosure of Interests: Chihiro Imaizumi: None declared, Yuki Inoue: None declared, Takaharu Kagtagiri: None declared, Sayaka Takenaka: None declared, Hideki Ito: None declared, Ayako Hirata: None declared, Takehisa Ogura: None declared, Hideto Kameda Grant/research support from: AbbVie, Asahi Kasei Pharma, Astellas, Chugai, Eisai, GlaxoSmithKlein, Mitsubishi-Tanabe, Novartis, Consultant for: AbbVie, Eli Lilly, Novartis, Speakers bureau: AbbVie, Asahi Kasei Pharma, Bristol-Myers, Chugai, Eli Lilly, Janssen, Mitsubishi-Tanabe, Novartis, Pfize DOI: 10.1136/annrheumdis-2019-eular.5327 